\title{
Lipochitin Oligosaccharides from Rhizobium leguminosarum bv. viciae Reduce Auxin Transport Capacity in Vicia sativa subsp. nigra Roots
}

\author{
Kees J. M. Boot, Anton A. N. van Brussel, Teun Tak, Herman P. Spaink, and Jan W. Kijne \\ Institute of Molecular Plant Sciences, Clusius Laboratory, Leiden University, Wassenaarseweg 64, 2333 AL \\ Leiden, The Netherlands \\ Accepted 8 July 1999.
}

Induction of the formation of root nodule primordia in legume roots by symbiotic rhizobia is probably preceded by a change in plant hormone physiology. We used a Vicia sativa (vetch) split root system to study the effect of inoculation with rhizobia or purified Nod factors (lipochitin oligosaccharides, LCOs) on polar auxin transport in roots. Addition of $R$. leguminosarum bv. viciae, the infective symbiote of vetch, to roots of its host plant reduced polar auxin transport capacity of these roots within $24 \mathrm{~h}$, in contrast to addition of non-nodulating, Sym plasmid-cured rhizobia. Addition of purified vetch-specific LCOs (NodRIv-IV/V[18:4,Ac]) caused a transient reduction in as little as $4 \mathrm{~h}$ after application, while after $16 \mathrm{~h}$ a second, stronger, and prolonged inhibition was observed that lasted at least $48 \mathrm{~h}$. This reduction of auxin transport capacity was in the same order of magnitude as inhibition by $N$-(1-naphthyl)phthalamic acid (NPA). Purified LCOs (NodRm-IV[16:2,Ac,S]) from Sinorhizobium meliloti, the symbiote of alfalfa, and chitopentaose were inactive, which indicates a specific effect of LCOs produced by $R$. leguminosarum bv. viciae. Auxin transport inhibition was restricted to the apical nodulation-susceptible part of the roots, whereas the upper parts of the roots showed no difference in auxin transport after treatment. The effect could be observed with as low as $10^{-9}$ M NodRlvIV/V[18:4,Ac] LCOs. Reduction of auxin transport by LCOs could not be inhibited by nitrate. Since inhibition of auxin transport capacity preceded the first root cortical cell divisions that result in root primordium formation, our results suggest a direct relationship between LCOs, polar auxin transport, and root nodule initiation, consistent with the hypothesis of U. Mathesius, H. R. M. Schlaman, H. P. Spaink, C. Sautter, B. G. Rolfe, and M. A. Djordjevic (Plant J. 14:23-34, 1998). However, nonmitogenic NodRIv-IV/V[18:1,Ac] showed a similar effect, which suggests that mitogenicity results from additional effects, in concert with auxin transport inhibition.

Under conditions of nitrogen deficit in the rhizosphere, (Brady/Sino/Meso/Azo) Rhizobium spp. bacteria can trigger

Corresponding author: Kees J. M. Boot; Fax: 31-71-5274999; E-mail: BOOT@RULBIM.LEIDENUNIV.NL the formation of nitrogen-fixing nodules in the roots of leguminous plants. This symbiotic interaction occurs in a hostspecific way (for recent reviews, see Dénarié et al. 1996; Heidstra and Bisseling 1996; Spaink 1996). For example, $R$. leguminosarum bv. viciae nodulates roots of pea and vetch, whereas $S$. meliloti can invade the roots of alfalfa and sweet clover plants. Nod factors (lipochitin oligosaccharides, LCOs) synthesized by the rhizobia are key determinants of this recognition process. The chemical structures of these LCOs differ between rhizobial species or biovars (Long 1996; Spaink 1996; Kamst et al. 1998). A major Nod factor of $S$. meliloti, NodRm-IV[16:2,Ac,S], differs from that of $R$. leguminosarum bv. viciae, NodRlv-V[18:4,Ac], in the nature of the fatty acyl side chain, in the $\mathrm{O}$-substitution of the reducing sugar residue, and in the number of $N$-acetyl-D-glucosamine residues. These differences can dramatically influence the response of different plant species toward LCOs. For example, vetch roots do not recognize LCOs produced by $S$. meliloti and do not respond to these Nod factors, although the oligochitin backbone of these LCOs is essentially the same as that of vetch-specific LCOs.

Primary target cells for LCOs are root epidermal cells producing a root hair. Among the first responses of these cells to rhizobia or to purified LCOs are membrane depolarization, intracellular alkalinization, calcium spiking, gene expression, and root hair deformation (Spaink 1996, and references therein). In addition, certain root cortical cells respond with mitotic activity, resulting in formation of a root nodule primordium. Purified LCOs from $S$. meliloti can induce formation of complete nodule structures on alfalfa roots (Truchet et al. 1991), whereas Nod factors produced by $R$. leguminosarum bv. viciae give rise to a limited number of cell divisions in the inner cortex of vetch roots (Spaink et al. 1991). Since formation of nodulelike structures in certain legume roots can be induced by auxin transport inhibitors such as $N$-(1-naphthyl) phthalamic acid (NPA) (Hirsch et al. 1989; Wu et al. 1996), it has been hypothesized that formation of nodule primordia is preceded by a change in plant hormone physiology (Hirsch and Fang 1994; Hirsch et al. 1997).

Recently, Mathesius et al. (1998) showed that LCOs from $R$. leguminosarum bv. trifolii can modify the expression pattern of an auxin-responsive reporter construct in roots of transgenic white clover plants. The authors suggested that LCOs act by perturbing the auxin flow in the root during the 
earliest stages of nodule formation. Two distinct auxin transport streams have been identified in (bean) roots: (i) a basipetal transport through root epidermal tissues, observed after microautoradiography, and (ii) an acropetal polar auxin transport (PAT) through the root central cylinder (Tsurumi and Ohwaki 1978). Auxin is the only plant hormone for which a polar transport has been demonstrated. PAT plays an important role in the normal growth and development of plants, such as in embryogenesis, gravitropism, phototropism, elongation growth, and lateral root development (Lomax et al. 1995). Therefore, it may likely play a role in root nodule formation. Recently, we found that for cortical explants of pea roots addition of the natural auxin indole acetic acid (IAA) was essential and sufficient for induction of cell divisions (K. J. M. Boot, unpublished results). In the present study, we measured polar transport of IAA in vetch roots after incubation with host-specific or mutant Rhizobium strains, as well as after addition of purified LCOs. We found that upon addition of hostspecific rhizobia or LCOs, the capacity of IAA transport in challenged roots was strongly reduced. A role for inhibition of PAT with respect to induction of root cortical cell divisions and nodulation is discussed.

\section{RESULTS}

\section{Auxin transport in Vicia sativa roots.}

As a test system, we used a split root system from Vicia sativa (vetch) plants. This system has the advantage that after treatment of one of the roots $(+$ root $)$, the other root (- root) can be used as a control.

First, we tested the ability of vetch roots to transport radioactively labeled indole 3 -acetic acid ( ${ }^{3} \mathrm{H}$-IAA) in the polar direction (basal to apical). During standard incubation of vetch root segments for $3 \mathrm{~h}$, IAA appeared to be transported from

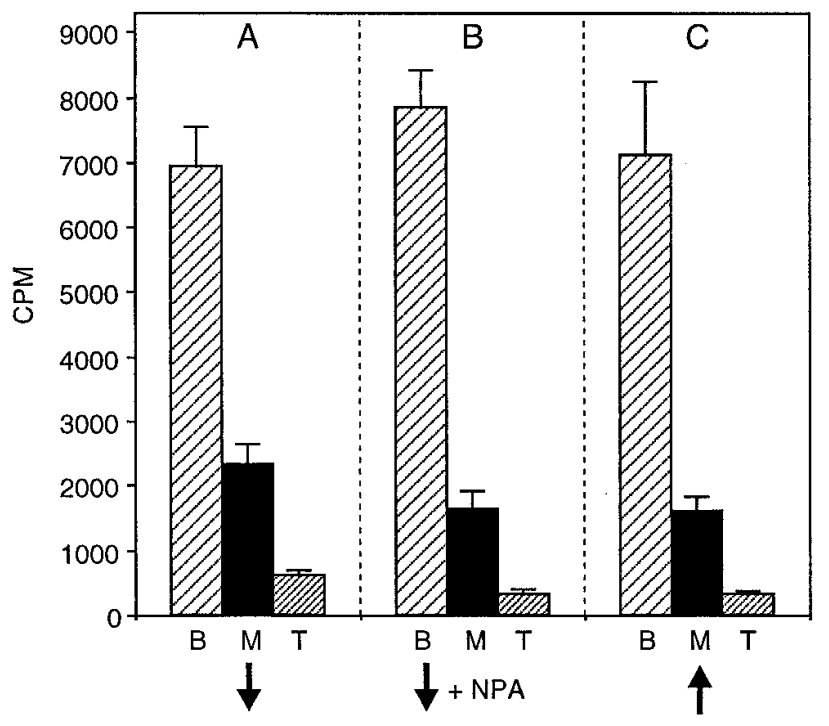

Fig. 1. Measurement of auxin transport capacity of Vicia sativa roots. Transport of indole 3 -acetic acid $\left({ }^{3} \mathrm{H}-\mathrm{IAA}\right)$ was measured in isolated root segments placed in agar donor blocks containing $1.10^{-7} \mathrm{M}$ concenrations of labeled IAA in the (A) basal to apical direction $(\downarrow),(\mathbf{B})$ basal to apical direction $(\downarrow)$ in the presence of $2.10^{-6} \mathrm{M} \mathrm{N}$-(1-naphthyl)phthalamic acid (NPA), or (C) apical to basal direction $(\uparrow)$. B, M, and T = bottom, middle, and top parts of the roots, respectively. the agar donor block into the apical part of the root. Part B (bottom) was the part positioned in the agar block, while parts $\mathrm{M}$ (middle) and T (top) were the more distal parts of the root, protruding from the agar block. As can be seen in Figure 1A, the bottom part accumulated large amounts of ${ }^{3} \mathrm{H}$-IAA while the middle and top parts ( $\mathrm{M}$ and $\mathrm{T}$ ) contained progressively smaller amounts of radioactive IAA. As expected, we found no difference in IAA transport capacity between two untreated roots of the same plant (data not shown). After application of the well-known auxin transport inhibitor NPA at a final concentration of $2 \cdot 10^{-6} \mathrm{M}$ to the agar donor block, accumulation of IAA in parts $\mathrm{M}$ and $\mathrm{T}$ appeared to be reduced, with a simultaneous increase in the amount of labeled IAA in part B of the root segments (Fig. 1B). Measurements of the accumulation of IAA in vetch roots that were placed in agar blocks in the inverted position (apical to basal) (Fig. 1C) yielded an amount of ${ }^{3} \mathrm{H}$-IAA in parts $\mathrm{M}$ and $\mathrm{T}$ similar to that observed for NPA-treated "basal-to-apical" positioned roots (compare Figure $1 \mathrm{~B}$ and $\mathrm{C}$ ). Taken together, these data showed that (i) use of the vetch split root system allows measurement of polar acropetal IAA transport, and (ii) accumulation of IAA in root parts $\mathrm{M}$ and $\mathrm{T}$ is only partly due to PAT (approximately $30 \%$ ), with the remaining part most probably being caused by passive diffusion of labeled auxin.

In the following experiments, the amount of labeled IAA that accumulated in part $\mathrm{M}$ during the standard incubation period was used as a measure for the transport capacity of the roots.

\section{Effect of Rhizobium spp. on auxin transport.}

After addition of the nodulating $R$. leguminosarum bv. viciae strain 5601 to one of the roots, we measured the auxin transport capacity in both treated $(+)$ and untreated $(-)$ roots at $t=0,24$, and $48 \mathrm{~h}$. In a separate experiment, we inoculated

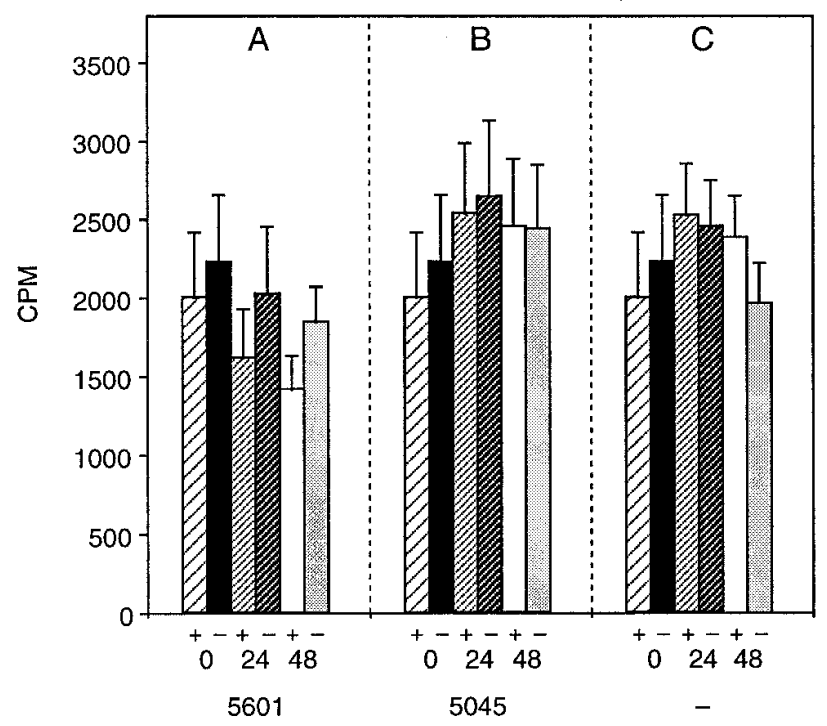

Fig. 2. Measurement of auxin transport capacity of $M$ (middle) parts of vetch roots after incubation with (A) Rhizobium leguminosarum bv. viciae 5601,which forms nodules on vetch roots, (B) R. leguminosarum bv. viciae 5045, a Sym plasmid-cured strain that does not produce lipochitin oligosaccharides (LCOs) and does not form root nodules on vetch roots, or (C) as a control, without rhizobia for the indicated time ( $t=0$, 24 , or $48 \mathrm{~h}$ ). $+=$ treated roots; $-=$ untreated roots. 
vetch roots with the non-nodulating strain 5045, a Sym plasmid-cured derivative of $R$. leguminosarum bv. viciae 5560 that is unable to produce LCOs. As a control, we used untreated roots. Figure 2 shows that after application of strain 5045 the IAA transport capacity of both + and - roots transiently increased to an extent similar to that in untreated control roots, whereas, by contrast, addition of strain 5601 caused a significant decrease in the auxin transport capacity of the + roots and to a lesser extent also of the - roots. The amount of accumulated labeled IAA in the + treated root segments after $48 \mathrm{~h}$ was close to the values found for NPA-treated roots and

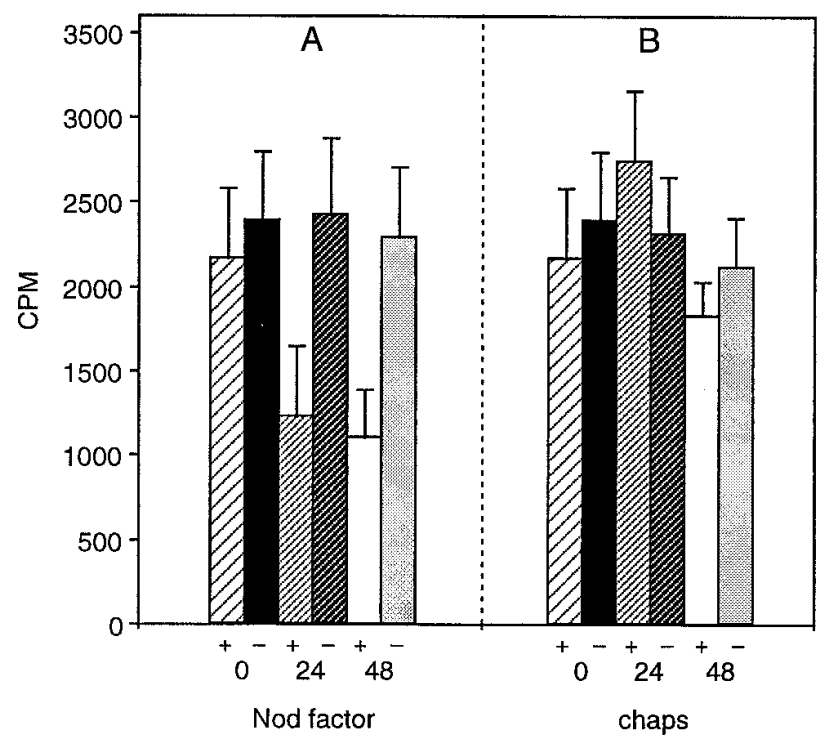

Fig. 3. Measurement of auxin transport capacity of $M$ (middle) parts of vetch roots after incubation with (A) lipochitin oligosaccharides (LCOs; NodRlv-IV/V[18:4,Ac]) at $10^{-7} \mathrm{M}$, which induce nodule primordia on vetch roots, or (B) with Chaps, a detergent used to dissolve the LCOs, as a control for indicated time $(t=0,24$, or $48 \mathrm{~h}) .+=$ treated roots; $-=$ untreated roots.

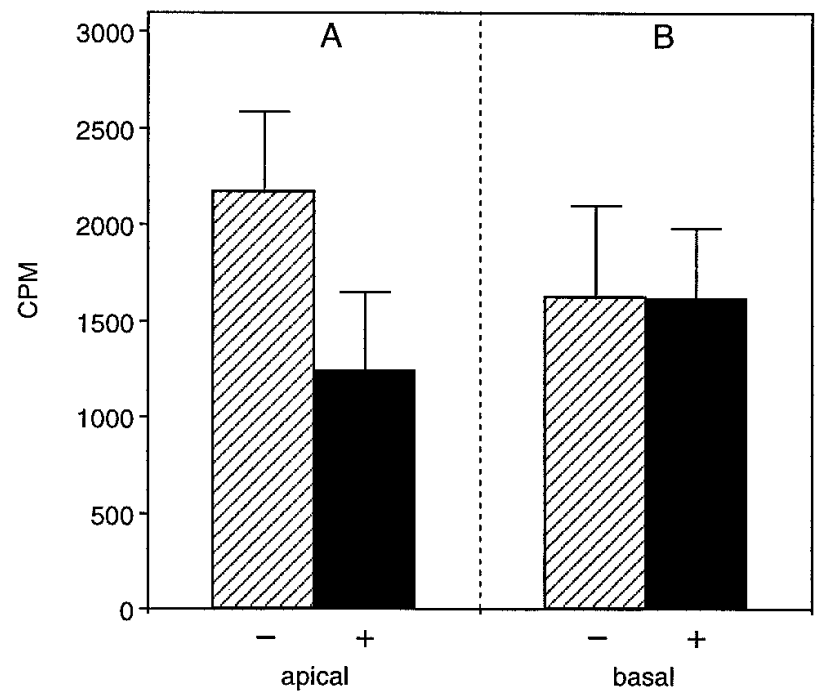

Fig. 4. Measurement of auxin transport capacity of $M$ (middle) parts of vetch roots at (A) apical or (B) basal part of the roots in the presence $(+)$ or absence (-) of lipochitin oligosaccharides (LCOs; NodRlv-IV/V $[18: 4, \mathrm{Ac}])$ at $10^{-7} \mathrm{M}$. Basal and apical parts obtained from same roots. for inverted root segments (compare Figures 1B, 1C, and 2). This result strongly suggested that rhizobial LCOs are responsible for the observed inhibitory effect.

\section{Effect of LCOs on auxin transport.}

Next, we tested the effect of addition of purified, vetchspecific LCOs (a mixture of NodRlv-IV[C18:4,Ac] and NodRlv-V[18:4]) to roots. Because these LCOs were dissolved in Chaps, this detergent was added to roots as a control. Application of $10^{-7} \mathrm{M}$ LCOs to roots of vetch plants resulted in a strong decrease of auxin transport capacity after only $24 \mathrm{~h}$, whereas added Chaps had no effect (Fig. 3). The amount of IAA that could be transported by the untreated (-) roots remained similar to that of control roots. Also after $48 \mathrm{~h}$, LCO-treated roots still showed strongly reduced auxin transport, whereas untreated roots responded normally.

Inhibition of the IAA transport capacity was specific for the apical parts of LCO-treated roots. Although (older) basal parts of treated roots had a lower auxin transport capacity than apical (younger) parts of the root, they showed no difference in auxin transport capacity following treatment with LCOs, compared with untreated control roots (Fig. 4). This result is consistent with the observation that apical root parts rather than basal root parts are susceptible to rhizobial infection. From these experiments we conclude that homologous rhizobia can locally inhibit the auxin transport capacity of their host plant roots through the action of LCOs.

Treatment of roots with LCOs and NPA together yielded results similar to those with LCOs alone (data not shown). This suggests that LCOs inhibit PAT rather than passive diffusion.

\section{Time course of auxin transport inhibition by rhizobia and LCOs.}

A time course of auxin transport measurements after root inoculation with $R$. leguminosarum bv. viciae 5601 revealed

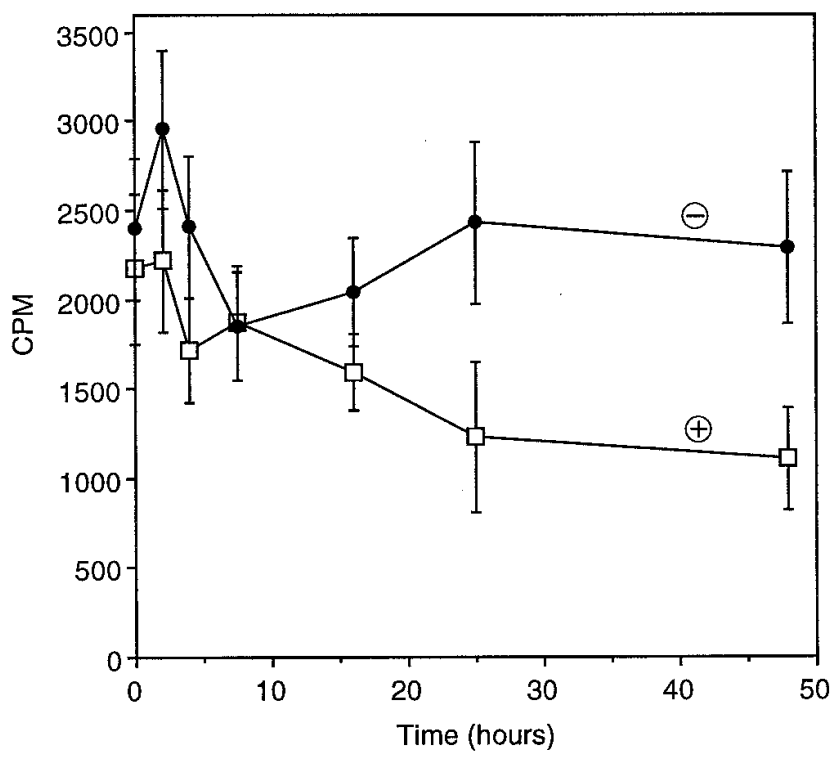

Fig. 5. Time course of changes in auxin transport capacity of $M$ (middle) parts of vetch roots where (A) the + roots were treated with lipochitin oligosaccharides (LCOs; NodRlv-IV/V[18:4,Ac]) at $10^{-7} \mathrm{M}$ and (B) the - roots were not treated. 
that these bacteria are able to diminish the auxin transport capacity of the inoculated roots only after an incubation period of $16 \mathrm{~h}$ (data not shown). However, treatment of roots with LCOs showed that these were able to significantly inhibit the auxin transport capacity in treated roots in as little as $4 \mathrm{~h}$ after

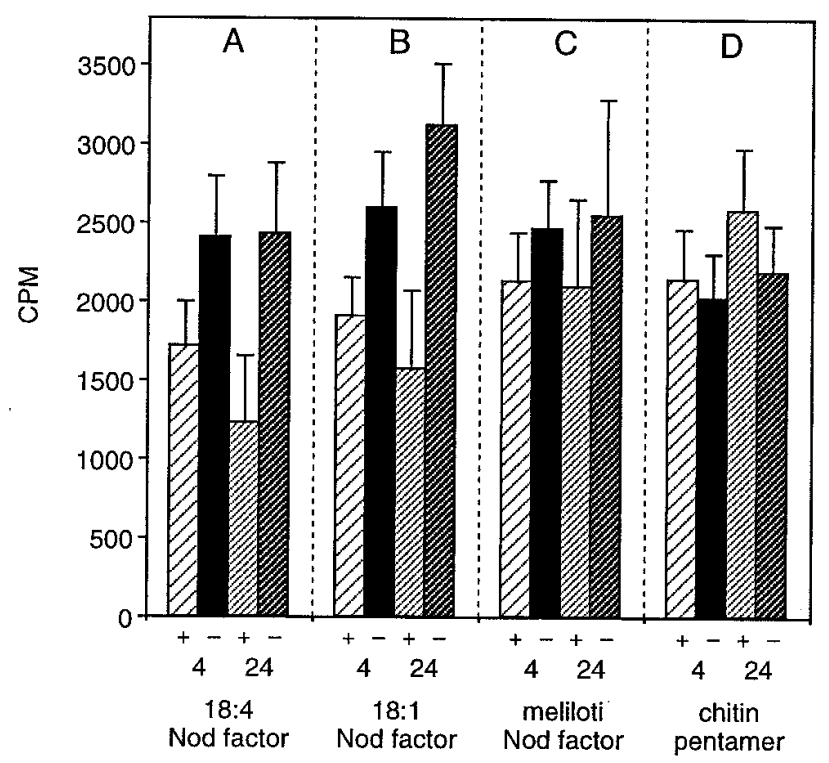

Fig. 6. Comparison of effects of different types of lipochitin oligosaccharides (LCOs) and chitin pentamers on auxin transport capacity of vetch roots. Polar auxin transport was measured in treated (+) and untreated (-) roots after 4 or $24 \mathrm{~h}$ of treatment of the + roots with (A) $10^{-7}$ M NodRlv-IV/V[18:4,Ac], (B) $10^{-7} \mathrm{M}$ NodRlv-IV/V[18:1,Ac], (C) $10^{-7}$ M NodRm-IV[16:2,Ac] produced by Sinorhizobium meliloti, which induces nodules on alfalfa, or (D) chitin pentamers at $10^{-6} \mathrm{M}$.

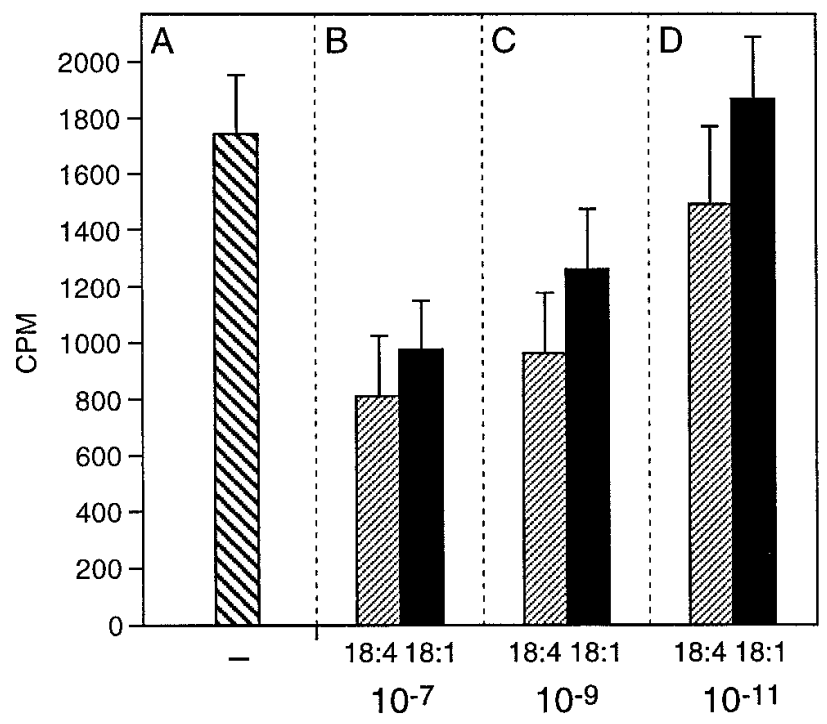

Fig. 7. Comparison of effects on polar auxin transport capacity vetch roots of different concentrations of lipochitin oligosaccharides (LCOs) NodRlv-IV/V[18:4,Ac] and NodRlv-IV/V[18:1,Ac] after treatment of + roots for $24 \mathrm{~h}$ with (B) $10^{-7} \mathrm{M}$, (C) $10^{-9} \mathrm{M}$, or (D) $10^{-11} \mathrm{M}$ of LCOs, compared with (A) untreated roots. Transport capacity of only the + roots is presented. Hatched bars represent C18:4-treated roots; filled bars C18:1-treated roots. application (Student's $t$ test, $P=0.02$; Fig. 5). The untreated roots first responded with a transient increase in auxin transport capacity that could be observed $2 \mathrm{~h}$ after application of LCOs, followed by an inhibition. Interestingly, for both the treated and untreated roots the initial moderate inhibition proved to be transient. While in untreated roots auxin transport capacity returned to control levels after $16 \mathrm{~h}$, the treated roots showed a second and prolonged inhibition of auxin transport between 8 and $16 \mathrm{~h}$ after application of LCOs. This resulted in a strong reduction of IAA transport capacity after 24 and $48 \mathrm{~h}$, as was already observed in earlier experiments.

\section{Specificity of inhibition of auxin transport by LCOs.}

Next, we tested the specificity of the auxin transport responses (after $4 \mathrm{~h}$ and after $24 \mathrm{~h}$ ) observed with LCOs. We used nonmitogenic LCOs from $R$. leguminosarum bv. viciae (a mixture of NodRlv-IV[C18:1,Ac] and NodRlv$\mathrm{V}[\mathrm{C} 18: 1, \mathrm{Ac}]$ ) at $10^{-7} \mathrm{M}$, LCOs from S. meliloti (NodRm-IV [C16:2,Ac,S), the symbiote of alfalfa, at $10^{-7} \mathrm{M}$, and chitin pentamers at $10^{-6} \mathrm{M}$ (Nod factors have an oligochitin backbone). Figure 6 shows that addition of alfalfa-specific LCOs or chitopentaose had no effect on auxin transport capacity after incubation of the roots for 4 and $24 \mathrm{~h}$. By contrast, addition of nonmitogenic LCOs (NodRlv-IV/V[C18:1,Ac]) of $R$. leguminosarum bv. viciae at $10^{-7} \mathrm{M}$ to roots yielded about the same response as did the mitogenic LCOs. After dilution, the mitogenic and nonmitogenic LCOs were still active at a concentration of $10^{-9} \mathrm{M}$, whereas at $10^{-11} \mathrm{M}$ they were no longer able to inhibit auxin transport (Fig. 7). In all experiments, mitogenic LCOs consistently showed a slightly greater activity than did nonmitogenic LCOs at the same concentration.

Effect of nitrate on inhibition of auxin transport by LCOs.

Since nitrate is a well-known inhibitor of nodulation (Malik et al. 1987), we were interested to see what effect nitrate would have on auxin transport capacity that is inhibited by LCOs. Therefore, we either precultured the plants on a me-

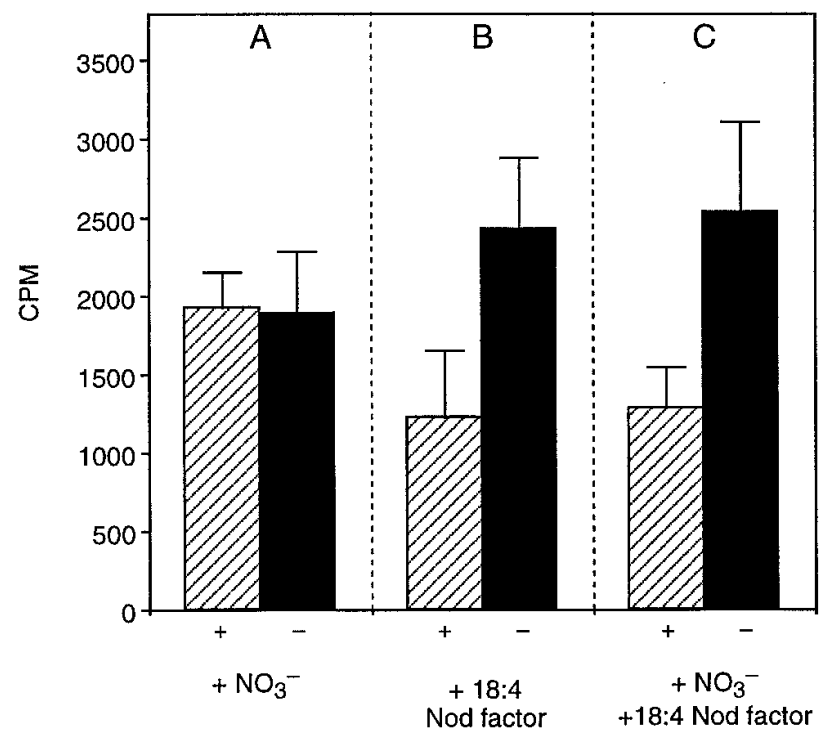

Fig. 8. Effect of nitrate on auxin transport capacity of vetch roots. Roots were treated (+) with (A) $20 \mathrm{mM}$ nitrate, (B) $10^{-7} \mathrm{M}$ NodRlvIV/V[18:4,Ac], or (C) both for $24 \mathrm{~h}$. 
dium containing $20 \mathrm{mM}$ nitrate for 3 days or simultaneously added this concentration of nitrate with LCOs, and we measured auxin transport $24 \mathrm{~h}$ after addition of the LCOs. As can be seen in Figure 8, simultaneous addition of nitrate and LCOs had no effect on the inhibition of auxin transport caused by the addition of LCOs, compared with the addition of LCOs alone. Addition of nitrate alone was without effect as well. Also, preculture of the plants on nitrate had no effect on the ability of LCOs to reduce the auxin transport capacity in roots (data not shown). While auxin transport inhibition by LCOs is an essential factor in root nodule formation, nitrate may affect the latter process at a later stage.

\section{DISCUSSION}

We have shown that LCOs produced by $R$. leguminosarum bv. viciae can inhibit PAT in vetch roots to a similar extent as does NPA. This reduction in auxin transport capacity is restricted to the apical nodulation-susceptible part of the roots, specific for LCOs from $R$. leguminosarum bv. viciae, even at concentrations as low as $10^{-9} \mathrm{M}$, cannot be inhibited by nitrate, and persists for at least $48 \mathrm{~h}$. A moderate transient reduction that can already be seen after $4 \mathrm{~h}$ is followed by a second, stronger response that starts between 8 and $16 \mathrm{~h}$ after addition of the LCOs.

These findings are in excellent agreement with the data published by Mathesius et al. (1998), who found that after spot inoculation of white clover roots with rhizobia or purified Nod factors from R. leguminosarum bv. trifolii the expression of an auxin-responsive GH3:GUS construct was downregulated during the first $30 \mathrm{~h}$. These authors explained this observation by assuming a local inhibition of auxin transport in the vascular bundle. Application of NPA induced the same changes in GH3:GUS expression as did LCOs or addition of $R$. leguminosarum bv. trifolii. Our results provide direct evidence for a local reduction of the auxin transport capacity in roots upon addition of homologous rhizobia or LCOs. Although we did not measure endogenous auxin transport but auxin transport capacity, it seems obvious to assume that these two are closely correlated.

In particular, the second. stronger, and longer-lasting response of vetch roots seems to be specific for $R$. leguminosarum bv. viciae-produced LCOs. This rhizobial biovar produces two types of LCOs, one carrying a special polyunsaturated fatty acid, NodRlv-IV/V[18:4,Ac], and another carrying a common fatty acid, NodRlv-IV/V[18:1,Ac]. Both factors are recognized by vetch roots and cause the "thick and short roots" (TSR) phenotype when roots are grown in the light in the absence of AVG (aminoethoxyvinylglycine), in contrast to LCOs from $S$. meliloti or chitopentaose, which do not yield any significant response in vetch roots. In addition, concentrations of $10^{-12}$ to $10^{-11} \mathrm{M}$ of $18: 4-\mathrm{LCO}$ or $18: 1$ LCOs were sufficient to induce root hair deformation within 3 $\mathrm{h}$ after addition to vetch roots. Although both types of LCOs are recognized, only NodRlv-IV/V[18:4,Ac] LCOs induce formation of root nodule primordia under standard conditions. Purified NodRlv-IV/V [18:1,Ac] LCOs are normally not mitogenic (Spaink et al. 1991). However, rhizobia mutated in their nodE gene, which produce only the latter type of LCOs, are able to induce the formation of nodules on vetch roots. Therefore, these types of LCOs are essentially capable of in- ducing cortical cell divisions in vetch roots, provided that they are produced by live bacteria. Most probably, the function of the live bacteria is the production of NodO, a nodulation protein that forms cation-selective channels in artificial bilayers and may enhance the biological activity of 18:1-LCOs (Sutton et al. 1994) In the absence of NodO, nod $\mathrm{E}$ mutants of $R$. leguminosarum bv. viciae are unable to nodulate pea and vetch (Downie and Surin 1990).

Recently, it was found that overexpression of ENOD40, a nodulation gene that is induced by specific and less-specific LCOs (Minami et al. 1996), results in an induction of cortical cell divisions in alfalfa roots (Charon et al. 1997). It has also been shown that differences exist in the expression patterns of ENOD12 and ENOD40 genes after addition of mitogenic or nonmitogenic LCOs (Horvath et al. 1993; Minami et al. 1996). For example, nonmitogenic Nod factors containing C18:1 fatty acid chains induced ENOD12 expression in pea root hairs after $24 \mathrm{~h}$, whereas the mitogenic LCOs containing a C18:4 acyl group showed expression after as little as $12 \mathrm{~h}$. Also, for ENOD 40, it has been shown that nonmitogenic Nod factors can induce gene expression but only transiently and in the pericycle of soybean roots, whereas mitogenic LCOs were capable of induction of prolonged gene expression that was also observed in dividing cortical cells. Apparently, timing and place of expression of nodulation genes are different for C18:4 and C18:1 types of LCOs, which can be important for establishment of a nodule meristem. Interestingly, a difference in timing between 18:4-LCO and 18:1-LCO activities was not apparent with respect to inhibition of auxin transport capacity.

Nitrate, a well-known inhibitor of nodule formation (Malik et al. 1987), inhibits root hair deformation, infection thread formation, and cortical cell division, but it did not inhibit the inhibition of auxin transport by vetch-specific LCOs. This suggests that nitrate inhibits auxin-induced cell division rather than auxin accumulation. Experiments with pea root cortical explants in which $20 \mathrm{mM}$ nitrate inhibited auxin-induced cell division corroborate this hypothesis (K. J. M. Boot, unpublished results).

Since the inhibition of auxin transport capacity precedes the first cortical cell divisions leading to nodule primordium formation, the mitogenic LCOs must have an additional effect that enables primordium formation. Still, auxin accumulation may be an essential prerequisite for this process to occur. Possible candidates for involvement in mitogenicity are sugars, cytokinins, or uridine (Bauer et al. 1996; Cooper and Long 1994; Smit et al. 1995).

\section{MATERIALS AND METHODS}

\section{Plant cultures.}

Vicia sativa L. subsp. nigra seeds were surface sterilized and germinated as described by van Brussel et al. (1982). The root tips from seedlings with 1 - to $1.5-\mathrm{cm}$-long roots were removed and the plants were then further cultivated in tubes on stainless steel grids with the roots suspended in Jensen medium (Vincent 1970) in a growth cabinet at $20^{\circ} \mathrm{C}$ with $120 \mu \mathrm{E}$ $\mathrm{s}^{-1} \mathrm{~m}^{-2}$ on the table surface (Philips TLD 50w/83HF tubes; day/night rhythm, $16 \mathrm{~h}$ light and $8 \mathrm{~h}$ darkness). After 7 days of growth, when lateral roots about $3 \mathrm{~cm}$ long had been formed, the plants were transferred to tubes containing little tubes (1 $\mathrm{cm}$ diameter) containing $5 \mathrm{ml}$ of Jensen medium. Each of two 
lateral roots with equal length was incubated in a different tube. The other roots were left hanging in the air around the little tubes. In this way, spatially separated roots A and B were further cultured for 3 days under the same conditions before addition of rhizobia or LCOs. All treatments were made under sterile conditions. To prevent stress caused by the presence of rhizobia or LCOs, AVG was routinely added to the roots at a final concentration of $0.1 \mathrm{mg} / \mathrm{ml}$ at the time of inoculation.

\section{Bacterial strains and chemicals.}

Rhizobium strains used in this study were R. leguminosarum bv. viciae RBL 5601 (Wijffelman et al. 1985) and LPR 5045 (Hooykaas et al. 1981). The NodRlvIV/V[18:4,Ac] and NodRlv-IV/V[18:1,Ac] LCOs produced by $R$. leguminosarum bv. viciae RBL 5601 were purified as described by Spaink et al. (1991). The appropriate concentrations were calculated after determination of the molar absorption coefficient of 18:4-LCOs and 18:1-LCOs, respectively. The $S$. meliloti LCOs NodRm-V[16:2,Ac] were a kind gift of G. Truchet. NPA was obtained from Pfaltz and Bauer (Waterbury, CT). ${ }^{3} \mathrm{H}$-IAA (specific activity $25 \mathrm{Ci} / \mathrm{mmol}$ ) was purchased from Amersham (Rosendaal, The Netherlands).

\section{Auxin transport assay.}

Transport of ${ }^{3} \mathrm{H}$-IAA was studied in 16-mm segments from vetch roots. The segments were cut $4 \mathrm{~mm}$ behind the root tip and were placed in agar blocks containing $1.10^{-7} \mathrm{M}$ labeled IAA, and AVG at a final concentration of $0.1 \mathrm{mg} / \mathrm{liter}$. The segments were incubated for $3 \mathrm{~h}$ in the dark at room temperature. To prevent drying, the roots were placed in silicon oil. After the incubation, root segments were cut in three pieces: part B (4 mm) was the fragment enclosed in the agar, and part $\mathrm{M}(4 \mathrm{~mm})$ and part $\mathrm{T}(8 \mathrm{~mm})$ were the middle and top parts of the root segments, respectively. Radioactivity in the segments was counted with a liquid scintillation counter. Each experiment was repeated at least three times with 6 or 12 plants per experiment.

\section{ACKNOWLEDGMENTS}

K. J. M. B. was supported by an NWO-pioneer grant awarded to H. P. S.

\section{LITERATURE CITED}

Bauer, P., Ratet, P., Crespi, M. D., Schultze, M., and Kondorosi, A. 1996. Nod factors and cytokinins induce similar cortical cell division, amyloplast deposition and MsEnod 12A expression patterns in alfalfa roots. Plant J. 10:91-105

Charon, C., Johansson, C., Kondorosi, E., Kondorosi, A., and Crespi, M. 1997. enod40 induces dedifferentiation and division of root cortical cells in legumes. Proc. Natl. Acad. Sci. USA 94:8901-8906.

Cooper, J. B., and Long, S. R. 1994. Morphogenetic rescue of Rhizobium meliloti nodulation mutants by trans-zeatin secretion. Plant Cell 6:215-225

Dénarié, J., Debellé, F., and Promé, J. C. 1996. Rhizobium lipochitiooligosaccharide nodulation factors: Signaling molecules mediating recognition and morphogenesis. Annu. Rev. Biochem. 65:503-535.

Downie, J. A., and Surin, B. P. 1990. Either of two nod gene loci can complement the nodulation defect of a nod deletion mutant of Rhizobium leguminosarum bv. viciae. Mol. Gen. Genet. 222:81-86.

Heidstra, R., and Bisseling, T. 1996. Nod factor-induced host responses and mechanisms of Nod factor perception. New Phytol. 133:25-43.

Hirsch, A. M., Bhuvaneswari, T. V., Torrey, J. G., and Bisseling, T. 1989. Early nodulin genes are induced in alfalfa root outgrowths elicited by auxin transport inhibitors. Proc. Natl. Acad. Sci. USA 86: 1244-1248.

Hirsch, A. M., and Fang, Y. 1994. Plant hormones and nodulation: What's the connection? Plant Mol. Biol. 26:5-9.

Hirsch, A.M., Fang, Y., Asad, S., and Kapulnik, Y. 1997. The role of phytohormones in plant-microbe symbiosis. Plant Soil 194:171-184.

Hooykaas, P. J. J., van Brussel, A. A. N., den Dulk-Ras, H., van Slogteren, G. M. S., and Schilperoort, R. A. 1981. Sym plasmid of Rhizobium trifolii expressed in different rhizobial species and Agrobacterium tumefaciens. Nature 291:351-353.

Horvath, B., Heidstra, R., Lados, M., Moerman, M., Spaink, H. P., Promé, J. C., Van Kammen, A., and Bisseling, T. 1993. Lipooligosaccharides of Rhizobium induce infection-related early nodulin gene expression in pea root hairs. Plant J. 4:727-733.

Kamst, E., Spaink, H. P., and Kafetzopoulos, D. 1998. Biosynthesis and secretion of rhizobial lipochitin-oligosaccharide signal molecules. Pages 29-71 in: Subcellular Biochemistry 29: Plant-Microbe Interactions. B. B. Biswas and H. K. Das, eds. Plenum Press, New York.

Long, S. R. 1996. Rhizobium symbiosis: Nod factors in perspective. Plant Cell 8:1885-1898.

Lomax, T. L., Muday, G. K., and Rubery, P. H. 1995. Auxin transport. Pages 509-530 in: Plant Hormones. P. J. Davies, ed. Kluwer Academic Pub., Dordrecht, The Netherlands.

Malik, N. S. A., Calvert, S. E., and Bauer, W. D. 1987. Nitrate induced regulation of nodule formation in soybean. Plant Physiol. 84:266-271.

Mathesius, U., Schlaman, H. R. M., Spaink, H. P., Sautter, C., Rolfe, B. G., and Djordjevic, M. A. 1998. Auxin transport inhibition precedes root nodule formation in white clover roots and is regulated by flavonoids and derivatives of chitin oligosaccharides. Plant J. 14:23-34.

Minami, E., Kouchi, H., Cohn, J. R., Ogawa, T., and Stacey, G. 1996. Expression of the early nodulin, ENOD40, in soybean roots in response to various lipo-chitin signal molecules. Plant J.10:23-32.

Smit, G., de Koster, C. C., Schripsema, J., Spaink, H. P., van Brussel, A. A., and Kijne, J. W. 1995. Uridine, a cell division factor in pea roots. Plant Mol. Biol. 29:869-873.

Spaink, H. P. 1996. Regulation of plant morphogenesis by lipo-chitin oligosaccharides. Crit. Rev. Plant Sci. 15:559-582.

Spaink, H. P., Sheely, D. M., van Brussel, A. A. N., Glushka, J., York, W. S,, Tak, T., Geiger, O., Kennedy, E. P., Reinhold, V. N., and Lugtenberg, B. J. J. 1991. A novel highly unsaturated fatty acid moiety of lipo-oligosaccharide signals determines host specificity of Rhizobium. Nature 354:125-130.

Sutton, J. M., Lea, E. J. A., and Downie, J. A. 1994. The nodulationsignaling protein NodO from Rhizobium leguminosarum biovar viciae forms ion channels in membranes. Proc. Natl. Acad. Sci. USA 91: 9990-9994.

Truchet, G., Roche, P., Lerouge, P., Vasse, J., Camut, S., de Billy, F., Promé, J. C., and Dénarié, J. 1991. Sulfated lipo-oligosaccharide signals of Rhizobium meliloti elicit root nodule organogenesis in alfalfa. Nature 351:670-673.

Tsurumi, S., and Ohwaki, Y. 1978. Transport of ${ }^{14} \mathrm{C}$-labeled indoleacetic acid in Vicia root segments. Plant Cell Physiol. 19:1195-1206.

van Brussel, A. A. N., Tak, T., Wetselaar, A., Pees, E., and Wijffelman, C. A. 1982. Small leguminosae as test plants for nodulation of Rhizobium leguminosarum and other Rhizobia and Agrobacteria harbouring a leguminosarum plasmid. Plant Sci. Lett. 27:317-325.

Vincent, J. M. 1970. A manual for the practical study of root-nodule bacteria. Pages 75-76 in: I. B. P. Handbook No. 15. Blackwell Scientific Pub., Oxford.

Wu, C., Dickstein, R., Carey, A. J., and Norris, J. H. 1996. The auxin transport inhibitor $N$-(1-naphthyl)phthalamic acid elicits pseudonodules on nonnodulating mutants of white sweetclover. Plant Physiol. 110:501-510.

Wijffelman, C. A., Pees, E., van Brussel, A. A. N., Okker, R. J. H., and Lugtenberg, B. J. J. 1985. Genetic and functional analysis of the nodulation region of the Rhizobium leguminosarum Sym plasmid pRL1JI. Arch. Microbiol. 143:225-232. 